\title{
Caracterización de los estudiantes con rasgos de deficit atencional del grado octavo del Colegio Nuestra Señora de Fátima de Cúcuta para el diseño de una propuesta didáctica que contribuya en el incremento de la atención
}

Luis Fernando Mariño ${ }^{1}$, Edith Solanye López Daza², Angélica Paola Chávez Bautista ${ }^{3}$.

\section{Resumen}

El siguiente proyecto de carácter descriptivo consistió en la identificación de los estudiantes de los grados octavo A y octavo C que presentaron rasgos de Déficit de Atención del Colegio Nuestra Señora de Fátima de Cúcuta, con el fin de especificar y analizar las características de estos jóvenes a nivel académico, disciplinar e interpersonal y con base en ellas crear una propuesta didáctica que ofrezca a los maestros diversas pautas y actividades que pueden aplicar durante las clases para incrementar los niveles de atención de aquellos estudiantes que posean este problema de aprendizaje.

Palabras Claves: Déficit de atención, Caracterización, Juego y Recurso Digital.

\section{They summarize}

The following project of descriptive character it consisted of the identification of the students of the degrees eighth To and eighth $\mathrm{C}$ that presented features of Deficit of Attention of the College Our Lady of Fátima de Cúcuta, in order to specify and to analyze the characteristics of these young persons to academic level, to discipline and interpersonal and with base in them to create a didactic offer that offers to the teachers diverse guidelines and activities that they can apply during the classes to increase the levels of attention of those students who possess this problem of learning.

Key words: Deficit of attention, Characterization, Game and Digital Resource.

${ }^{1}$ Licenciado en Matemáticas y Computación - UFPS. Especialista en Computación para la Docencia - UAN. Magister en educación Matemática - UNET.E-mail: fermarin3@gmail.com

2Licenciada en Matemáticas e Informática - UFPS. E-mail: solanyelod@hotmail.com

32Licenciada en Matemáticas e Informática. - UFPS. E-mail: angiechavezbautista@ hotmail.com 


\section{Introducción}

$\mathrm{D}$ e acuerdo con lo establecido en la Ley General de Educación, el servicio educativo para aquellas personas con dificultades de aprendizaje es un aspecto muy importante que prevalece y requiere de una atención especial por parte de las instituciones de enseñanza, tanto que la ley exige que estas organicen acciones pedagógicas y terapéuticas pertinentes con las cuales se logre integrar académica y socialmente a todos los estudiantes afectados.

A partir de ello y gracias a las Prácticas Pedagógicas realizadas por las investigadoras en el Colegio Nuestra Señora de Fátima nació la iniciativa de con este proyecto analizar y proponer soluciones a una de las causas de dificultades de aprendizaje más significativa y compleja observada: los rasgos de déficit de atención (DDA) en algunos estudiantes.

Se encontró que calificar el DDA como un problema a nivel educativo es identificarlo como un trastorno que hace referencia a la alteración causada por la carencia, ausencia e insuficiencia de actividades de orientación, selección y mantenimiento de la atención: lo cual crea como consecuencia una deficiencia en el control y regulación en el proceso de enseñanza y aprendizaje, es decir, una problemática compleja en la que juega un papel principal la acción de la Institución Educativa, y más específicamente del maestro [1].

No obstante, con este trabajo no se pretendió establecer si un estudiante posee DDA, pues está fuera del alcance de la Institución Educativa, por tal motivo lo que se hizo fue describir a nivel académico, disciplinar, e interpersonal a aquellos estudiantes en quienes se identificaron rasgos de este trastorno, y cuando se tuvo un mayor conocimiento de su dificultad se seleccionaron estrategias didácticas que contribuyen en incrementar su atención, con las cuales se diseñó una cartilla llamada "El juego como estrategia didáctica para estudiantes desatentos".

\section{Cuerpo del trabajo}

Todo inició con el diagnóstico, esta fue la fase en la que se indagó si en realidad existían casos de rasgos de DDA en los grados $8^{\circ} \mathrm{A}$ y $8^{\circ} \mathrm{C}$, y este objetivo se logró gracias a tres acciones: la aplicación de una escala de valoración de DDA, de unas pruebas y de una encuesta a algunos profesores de la Institución Educativa.

La primera en utilizar fue la Escala de Valoración de DDA desarrollada por la APA [2] en el Manual DSM-IV [3], ésta consiste en nueve criterios de los cuales un joven para que le sea diagnosticado DDA debe haber cumplido por lo menos durante seis meses con seis o más de estos síntomas, lo que se hizo fue analizar en cada uno de los 58 estudiantes de $8^{\circ} \mathrm{A}$ y $8^{\circ} \mathrm{C}$ (muestra de la investigación) estos nueve aspectos y de esto surgió que en el curso $8^{\circ} \mathrm{A}$ conformado por 28 estudiantes el $17.9 \%$, de ellos y en el grado $8^{\circ} \mathrm{C}$ constituido por 30 estudiantes el $23.3 \%$ de ellos obtuvo una valoración desfavorable, ya que cumplieron con 6 o más de los criterios establecidos.

Con el fin de corroborar los resultados anteriores se aplicaron una serie de pruebas, exactamente siete ejercicios que evalúan la atención, los cuales desarrollaron los estudiantes individualmente durante las horas de clase y las cuatro iniciales en un tiempo máximo de cinco minutos, la primera consistió en una ficha en la cual debían encontrar las 15 diferencias que existen entre dos imágenes aparentemente iguales, la segunda en una ficha en la que debían marcar en unos cuadros de letras las columnas y filas en las habían letras repetidas, la tercera fue una sopa de letras en la que debían descubrir los nombres de doce colores, la cuarta correspondió a seis ejercicios de razonamiento lógico, la quinta y sexta fueron dos juegos de memoria de la página web "Ibraining", y la séptima consistió en un rompecabezas de 36 piezas.

Estas actividades comenzaron desarrollándolas los 58 estudiantes pero cada dos pruebas se fueron excluyendo aquellos jóvenes que obtenían en ambas un puntaje inferior al $60 \%$, es así como, luego de realizar las pruebas 1 y 2 se hizo la primera selección de estudiantes y de acuerdo al desempeño continuaron en el curso $8^{\circ} \mathrm{A} 17$ estudiantes y en el curso $8^{\circ} \mathrm{C} 19$ estudiantes. 
Luego se realizó la segunda selección al finalizar las pruebas 3 y 4 , en este caso siguieron por el grado $8^{\circ} \mathrm{A} 10$ estudiantes y por $8^{\circ} \mathrm{C} 9$ estudiantes.

La tercera selección se efectuó al terminar las pruebas 5 y 6, como estos eran juegos en línea no hubo un tiempo límite para realizarlos ya que el mismo juego proporcionó el puntaje final, continuaron 7 estudiantes de cada uno de los cursos.

Y para la última selección, luego de la prueba siete, se cronometró el tiempo que tardaron armando el rompecabezas y respecto a lo obtenido continuaron 3 estudiantes de $8^{\circ} \mathrm{A}$ y de $8^{\circ} \mathrm{C}$.

Es decir, estos seis jóvenes según lo que evaluaban estos ejercicios tienen dificultades en su atención, y así al obtenerlos se prosiguió a compararlos con los obtenidos anteriormente en la Escala de Valoración, y se encontró que en efecto estos seis estudiantes coincidían.

De esta manera, se concluyó que tres estudiantes del grado $8^{\circ} \mathrm{A}$ y 3 estudiantes del grado $8^{\circ} \mathrm{C}$ de la Institución Educativa Nuestra Señora de Fátima de Cúcuta presentan rasgos de Déficit de Atención.

Se debía responder sobre un estudiante en particular entonces se ordenó de manera que dos profesores la contestaran acerca de un mismo estudiante, en consecuencia de los seis estudiantes pertenecientes al proyecto surgieron 12 encuestas diferentes.

Como última medida de diagnóstico se utilizó una encuesta a 12 profesores de la Institución Educativa, con el objetivo de conocer, analizar y cerciorar su opinión acerca del rendimiento académico, comportamiento y desarrollo socioemocional de cada uno de los seis estudiantes seleccionados en el proyecto.

Esta encuesta consistió en siete preguntas de respuesta abierta y una de opción múltiple con múltiple respuesta, se organizó en tres aspectos: académico, disciplinar e interpersonal, y fue elaborada teniendo en cuenta las características a interpretar y analizar en estos estudiantes que presentan bajo nivel de atención. Este instrumento se aplicó en las horas libres de los profesores y en iguales condiciones.
Habiendo ya terminado la primera fase del proyecto de investigación se continuó a analizar con más detalle a estos seis estudiantes, lo que se hizo fue precisar las características en el aspecto académico, disciplinar e interpersonal de cada uno de los jóvenes, comparar dichas características, considerar las comunes y razonar especialmente sobre ellas. De este análisis se encontraron efectivamente nueve características con las cuales se determinó la caracterización final de los estudiantes con rasgos de DDA de octavo grado del Colegio Nuestra Señora de Fátima, además al cotejar estas características con las propias de DDA resultaron ser muy similares.

\section{Propuesta}

Con el fin de elaborar una propuesta didáctica que contribuya a mejorar los niveles de atención de los estudiantes de octavo grado que presentan rasgos de déficit de atención, se realizó lo siguiente:

1. Se examinaron, seleccionaron y analizaron las estrategias didácticas más adecuadas que contribuyen a aumentar los niveles de atención en los estudiantes, dichas estrategias didácticas tuvieron dos enfoques: juego, y recurso digital.

2. Se realizó una prueba piloto de tres de estas estrategias con los estudiantes que presentan rasgos de DDA con el propósito de evaluar la factibilidad y efectividad de la propuesta didáctica.

3. Las anteriores estrategias junto con una serie de información y pautas acerca del DDA se recopilaron en una cartilla.

4. Como resultado de la propuesta surgió la cartilla "El juego como estrategia didáctica para estudiantes desatentos".

La cartilla cuenta con una estructura especial que consta de dos partes: Las Pautas y Las Actividades, la primera consiste en diversas pautas para intervenciones educacionales y la segunda consta de diferentes estrategias organizadas en dos tipos: juegos y recurso digital, ambas útiles para incentivar a los estudiantes y reforzar su memoria y concentración. 


\section{Resultados y Discusión}

De acuerdo al análisis realizado de las fases llevadas a cabo durante el desarrollo del proceso investigativo, se muestra a continuación los resultados obtenidos en el cumplimiento de cada uno de los objetivos planteados:

Teniendo en cuenta el primer objetivo del proyecto el cual fue identificar los estudiantes del grado octavo que presenten rasgos de déficit de atención; y las actividades realizadas para lograrlo se obtuvo en cada acción:

Según los resultados de la escala de valoración de DDA del manual DSM-IV y lo establecido por la APA, se puede afirmar que el 17,9\% de los estudiantes del grupo de $8^{\circ} \mathrm{A}$ que corresponde a 5 de ellos y el $23,4 \%$ del grado $8^{\circ} \mathrm{C}$, es decir, 7 estudiantes podrían presentar DDA.

Lo anterior indica que los criterios o síntomas que más presentan estos estudiantes son: a menudo no presta atención suficiente a los detalles o incurre en errores por descuido en las tareas escolares y a menudo se distrae fácilmente por estímulos irrelevantes; con el 100\% cada uno. El criterio o síntoma que menos presentan estos estudiantes es: a menudo parece no escuchar cuando se le habla directamente, con un 33\%.

Según el proceso de selección de los estudiantes de acuerdo al desempeño en las 7 pruebas se obtuvo que 3 estudiantes del grado $8^{\circ}$ a y 3 estudiantes del grado $8^{\circ} \mathrm{C}$ presenten dificultades de atención.

En cuanto a los resultados de la información obtenida de la encuesta realizada a algunos maestros de la institución educativa sobre el aspecto académico, disciplinar e interpersonal de los estudiantes en quienes se identificó rasgos de déficit de atención, indican que la mayoría de ellos poseen un rendimiento académico medio, su ritmo de aprendizaje es lento, no tienen una estrategia de estudio, muy poco se mantienen atentos a las clases, ante sus elogios, regaños o castigos son tolerantes y aceptan con respeto, además mantienen buen trato con sus compañeros.

Con el propósito de cumplir el segundo objetivo de este proyecto, el cual fue analizar y comparar las características comunes de los estudiantes con rasgos de déficit de atención durante su proceso de aprendizaje, se realizó una caracterización individual y una final según el aspecto académico, disciplinar e interpersonal de los estudiantes seleccionados durante la fase de diagnóstico: su atención es dispersa, su ritmo de aprendizaje en la mayoría de los casos es lento, tienen dificultades para memorizar, cometen errores por descuido, a menudo dejan sus labores escolares incompletas, son desordenados en sus apuntes, son poco responsables con sus deberes escolares, se les dificulta abstraer la información importante y seguir instrucciones, tienen una personalidad pasiva; pero en ocasiones se comportan de manera impulsiva y en la mayoría de los casos tiene un grupo de amigos.

Con el fin de lograr el tercer y último objetivo de este proyecto el cual era elaborar una propuesta didáctica que contribuya a mejorar los niveles de atención de los estudiantes de octavo grado que presentan rasgos de déficit de atención, se diseñó la cartilla "El juego como estrategia didáctica para estudiantes desatentos". La cual según la prueba piloto que se realizó, resulto interesante y llamativa para los maestros y ya que la concibieron como una oportunidad para apropiarse de conocimientos teóricos, prácticos y didácticos acerca del tema y así generar un ambiente adecuado en el aula de clase en el cual el estudiante con dificultades de atención fortalezca la adquisición de conocimientos, destrezas y competencias y así mismo aumente su nivel de atención y de igual forma para los estudiantes fue de gran interés y agrado.

\section{Conclusiones}

Luego de los resultados obtenidos en la investigación se concluyó que:

Los resultados del proceso de diagnóstico realizado a los estudiantes de los curso de $8^{\circ} \mathrm{A}$ y $8^{\circ} \mathrm{C}$ del Colegio Nuestra Señora de Fátima, permitió identificar en cada curso tres jóvenes con rasgos de déficit de atención.

Según el análisis realizado a los seis jóvenes participantes del proyecto de investigación se puede afirmar que no todos los estudiantes con rasgos de este trastorno manifiestan los mismos síntomas y con la misma intensidad, pero si existe una serie 
de comportamientos comunes entre ellos y que al compararlas con las cualidades representativas de los niños con déficit de atención resultaron ser muy similares.

Gracias a la prueba piloto de la propuesta didáctica planteada fue evidente que los juegos incluidos en esta son una excelente alternativa para tratar y ayudar aquellos estudiantes que presentan rasgos de Déficit Atencional, debido que además de estimular la capacidad de atención de los jóvenes, ejercitan su memoria, su razonamiento lógico, su organización y concentración en un solo objeto también se pueden desarrollar en cualquier ambiente de clase fomentando el aprendizaje de una temática en particular.

La aplicación informática Ardora resultó ser una excelente estrategia didáctica, ya que al contar con 34 actividades le permite al profesor crear fácilmente ejercicios de cualquier tema que exigen agilidad mental, atención visual, atención sonora, concentración, memoria, razonamiento lógico, y con los cuales el estudiante se divierte y aprende.

Un estudiante con rasgos de déficit de atención no está destinado al fracaso o a ser fuente de frustración, en realidad tienen altas probabilidades de alcanzar las metas académicas y sociales esperadas para su edad, si su energía y todas sus cualidades se canalizan correctamente.

\section{Bibliografía}

[1] LÓPEZ, M. E. Tareas sin Peleas. Bogotá: Círculo de Lectores. 2003

[2] Asociación Psiquiátrica Americana.

[3] Manual diagnóstico y estadístico de los trastornos mentales. Cuarta Edición 